\title{
Strategies of the treatment of spontaneous esophageal rupture: Report of thirteen cases from Xingtai, Hebei Province, China
}

\author{
Di Yonghui \\ Department of Thoracic Surgery, Xingtai People's Hospital, Xingtai, Hebei Province, 054001, China
}

\begin{abstract}
Aims and Objectives: To explore the treatment of spontaneous esophageal rupture. Materials and Methods: The treatments of thirteen patients hospitalized with spontaneous esophageal rupture were analyzed retrospectively. Initially three cases of hospitalized patients were given operative treatments. During the surgery, food residue and necrotic tissue were removed, and repair of esophageal rupture and jejunostomy were implemented. One closed chest drainage tube was placed at the end of the surgery. All the patients were given nutritional support through vein and nutrient canal placed into the jejunum. The remaining ten cases were given conservative treatments. Early treatments included closed chest drainage, and irrigation with normal saline by the patients' swallowing. At the same time were given intravenous nutrition. When the patients' conditions were improved, three cases were given nutrition through nasogastric tube at the same time. The remaining seven cases were given jejunostomy and were given nutritional support through vein and nutrient canal placed into the jejunum. Results: In the first 3 patients, who were given emergency operative treatments, two cases died, only single case $(33 \%)$ recovered. In the other ten cases, one case gave up treatment and discharged from hospital (died of infection with multiple organ failure after discharge). One case had encapsulated pyothorax with atelectasis after chest drainage, and the healing of esophagus rupture were influenced, so at the $25^{\text {th }}$ day after esophageal rupture, pyothorax removal and repair of esophageal rupture were implemented, and the patient was recovered well after surgery. The rest eight cases were recovered well after closed chest drainage treatment $(80 \%)$ without surgery. Conclusion: For the treatment of spontaneous esophageal rupture, we think that it should be given priority to conservative treatment. With adequate drainage and irrigation, and keeping balance of nutritional condition and water-electrolyte, a good therapeutic effect can usually be achieved.
\end{abstract}

Key words: Spontaneous esophageal rupture, Operative treatment, Conservative treatment

\section{INTRODUCTION}

Spontaneous esophageal rupture is one of the thoracic surgery emergencies, which were rare before. But in recent years the incidence of this disease is on the rise, and can be seen especially after drinking or satiety. The misdiagnosis rate is high, according to the report of $75 \%,{ }^{1}$ the mortality is still staying at a high level. The reasons may be are related to the poor recognition of people to the disease. The current article presents thirteen spontaneous esophageal rupture patients admitted in our department as follows, and hope to be able to help in the diagnosis and treatment of esophageal rupture.

\section{MATERIALS AND METHODS}

The current study was conducted from November 2010 to January 2014, thirteen cases were treated in our hospital due of spontaneous esophageal rupture were retrospectively analyzed. All patients were male, and were from 24 to 70 years old, the average age was 55.5 years. Eleven patients admitted to hospital complained for nausea and vomiting after drinking or satiety, with chest pain or discomfort behind the sternum. Two of the cases had no history of drinking or eating and with no obvious history of nausea and vomiting. Among them, seven cases complained chest 
tightness and shortness of breath, one case with abdominal pain, and one case with back pain. The treatment time was from 5 hours to 33 hours. Only one of the cases undergone surgery was conducted after twenty-four hours (33 hours) after consulting with the patient, remaining of the twelve cases undergone surgery which were within twenty-four hours after consultation.

All patients were advised of chest CT scan in emergency department before admitting in hospital. Hydropneumothorax were seen unilaterally or bilaterally in CT scan, with or without mediastinal emphysema. All the patients were diagnosed after admission by pleural puncture draining out brown gastric juice (Figure 1).

The treatment time of the first three patients was less than 24 hours, so they were given operative treatments. With a lateral thoracotomy, the esophageal ruptures were found in the lower esophagus, and about 5-12 cm long. During the surgery, food residue and necrotic tissue were removed, and repair of esophageal rupture and jejunostomy were implemented, and also closed chest drainage tubes were placed. All the patients were given nutritional support through vein and nutrient canal placed into the jejunum.

The remaining ten cases were given conservative treatments. Early treatments included closed chest drainage, and irrigation with normal saline by the patients' swallowing. At the same time were given intravenous nutrition. When the patients' conditions was improved, three of them were given nutrition through nasogastric tube at the same time. The remaining seven cases were given jejunostomy and were given nutritional support through vein and nutrient canal placed into the jejunum. All of the ten patients were performed upper gastrointestinal gastrografin radiography during hospitalization, and showed that all the esophageal ruptures located in the lower esophagus, of which one case was detected with breakage into both thoracic cavities.

\section{RESULTS}

In the first three patients, who were given emergency surgery treatments, two of them died and only one patient $(33 \%)$ recovered. Among the other ten patients, one of them gave up treatment and was discharged from hospital (died of infection with multiple organ failure after discharge). One of them had encapsulated pyothorax with atelectasis after chest drainage (Figure 2), and the healing of esophagus rupture were influenced, so at the $25^{\text {th }}$ day after esophageal rupture, pyothorax removal and repair of esophageal rupture were implemented, and the patient was recovered well after surgery. The rest of the eight patients had good recovery after closed chest drainage treatment (80\%) without surgery (Table 1 and Figure 3).

\section{DISCUSSION}

Spontaneous esophageal rupture is caused by various reasons which can suddenly increase the pressure in esophageal cavity, and full-thickness of the esophagus tear. It can also be called Boerhaave's syndrome, because it was first proposed by Boerhaave in 1724. The disease usually occurs in middle-aged man, and rarely in woman and child. ${ }^{2}$ Most of the patients presents with nausea, vomiting, and then appear esophageal rupture. It can also occur in woman during childbirth which can increase abdominal pressure. All the patients are men in this study, including four patient presented with vomiting after drinking, seven with vomiting after satiety and three patients with pain or discomfort behind sternum without significant inducements. The number of patients with vomiting accounted for $84.6 \%$ of the total number of patients, consistent with the literature reported proposal. ${ }^{3}$ The etiological factors associated with this disease is still not clear at present but, reports from previously conducted studies states that eosinophilic esophagitis is one of the causes of spontaneous esophageal rupture ${ }^{4,5}$ and states that eosinophilic esophagitis is a transmural disease, spontaneous esophageal rupture is a serious complication of eosinophilic esophagitis. Some of the studies reported it as anti-phospholipid antibody syndrome which can cause the disease recurrently. ${ }^{6}$ Based on esophagus dissection, in lower segment esophagus longitudinal muscle fibers are less, and smooth muscle is the main muscle fiber, so muscular layer is of less thickness. At the same time due to less support of muscles around the lower esophagus, this segment is more prone to rupture after exposure to the outside force, so the disease usually occurs in lower segment of esophagus.

In this study, all the patients were confirmed by surgery or radiography that all the ruptures of the esophagus located in the lower esophagus, consistent with the literature reported proposal. , $^{1,8}$

\section{Table 1: Treatment schedule of patients}

\begin{tabular}{|c|c|c|}
\hline Treatment methods & $\begin{array}{c}\text { Case } \\
\text { number }\end{array}$ & Prognosis \\
\hline $\begin{array}{l}\text { Repair of ruptured } \\
\text { esophagus }\end{array}$ & 3 & $\begin{array}{l}1 \text { Case recovered, } \\
2 \text { Cases died of chest } \\
\text { infection and multiple } \\
\text { organ failure }\end{array}$ \\
\hline $\begin{array}{l}\text { Closed chest drainage+ } \\
\text { nasal feeding }\end{array}$ & 3 & $\begin{array}{l}2 \text { Cases recovered, } \\
1 \text { Case gave up treatment }\end{array}$ \\
\hline $\begin{array}{l}\text { Closed chest drainage+ } \\
\text { jejunal nutrient }\end{array}$ & 7 & $\begin{array}{l}6 \text { Cases recovered, } \\
1 \text { Case had encapsulated } \\
\text { pyothorax, and was given } \\
\text { thoracotomy }\end{array}$ \\
\hline
\end{tabular}

Asian Journal of Medical Sciences | May-Jun 2016 | Vol 7 | Issue 3 


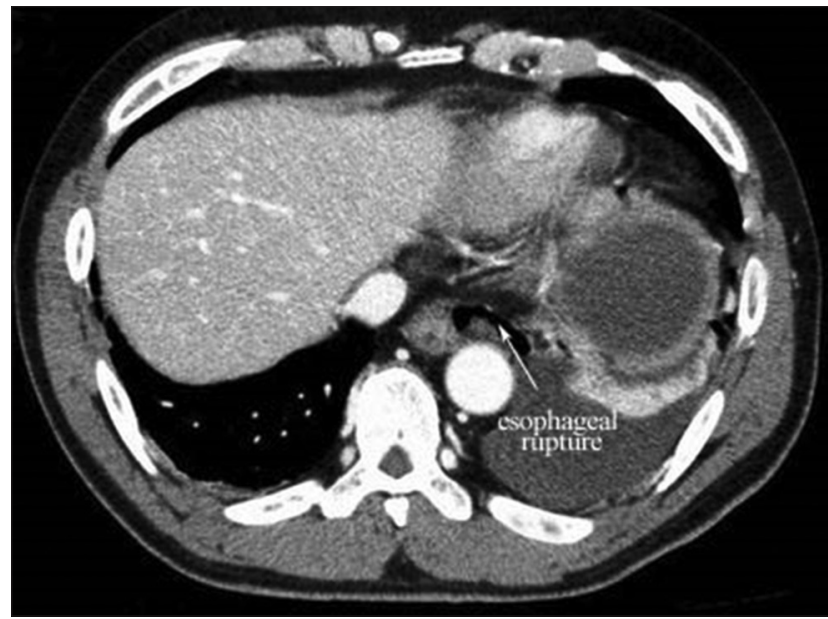

Figure 1: Esophageal rupture

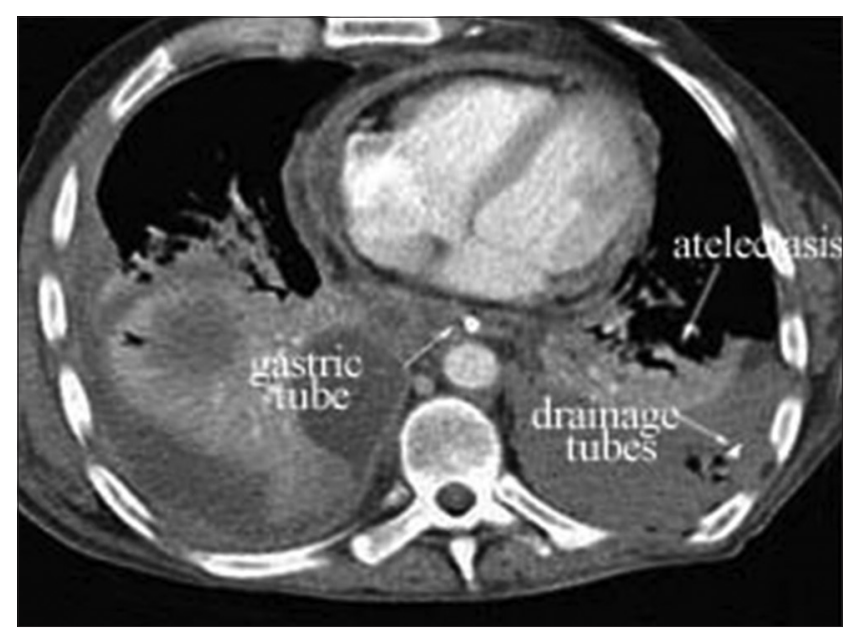

Figure 2: Encapsulated pyothorax with atelectasis

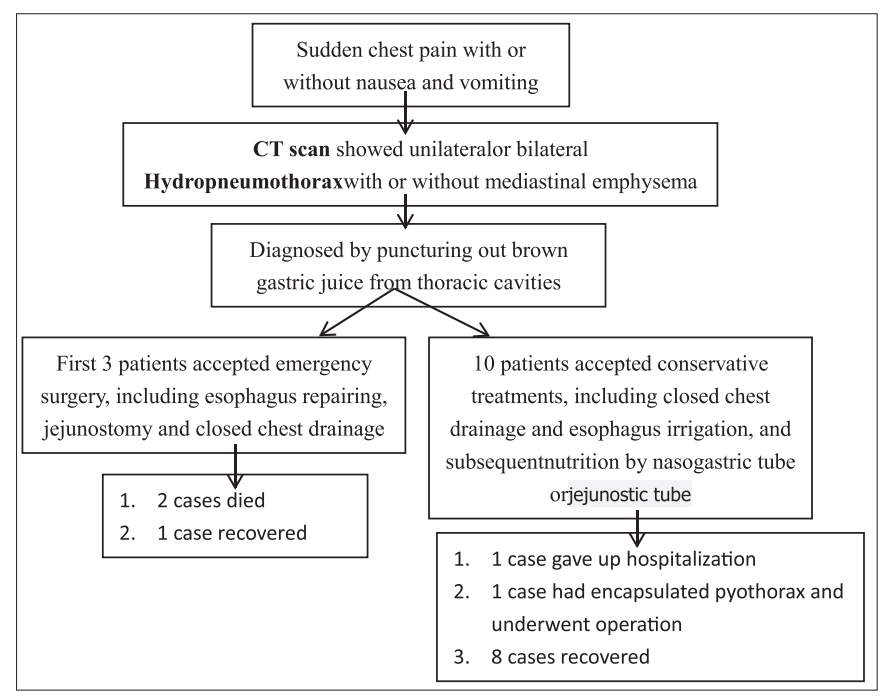

Figure 3: Treatment strategies

Spontaneous esophageal rupture is one of the serious emergencies of thoracic surgery department, and increase gradually due to higher incidence of alcohol consumption in the recent years. The disease is very dangerous, and with a delayed treatment time or misdiagnosis, it often goes to shock, hypoxemia, multiple organ failure, and even death. Because the clinical symptoms are often atypical in these patients, ${ }^{9}, 10,11$ misdiagnosis often be made, especially when patients does not presents with nausea and vomiting. If the patient complains of flustered, chest tightness, this disease may often be confused with heart disease, lung disease. ${ }^{12}$ Some patients also complains of abdominal pain, ${ }^{13}$ and can often be misdiagnosed as acute abdominal disease such as pancreatitis. So right diagnosis on time is very important for good prognosis and recovery. In this study, one patient had no symptoms of nausea and vomiting, only complained of discomfort behind sternum and chest tightness. In outpatient he was considered may be angina at first. Luckily the clinic doctor gave him chest CT scan besides the usual examination such as electrocardiogram (ECG) etc and made a right diagnosis. One patient complained of abdominal pain without discomfort symptoms of chest, he was found abnormality in the chest during an emergency abdominal X-ray, so a chest CT scan was performed. In fact, the diagnosis of this disease is not difficult. If there is a clear history of drinking or satiety, with a complaint of chest pain, chest tightness after a sudden vomiting, spontaneous esophageal rupture should be considered first. With an examination of chest CT scan, if pneumomediastinum and pleural effusion can be seen, further diagnosis can be made. If food residues can be seen in chest drainage fluid, or esophageal gastrografin radiography shows leakage from esophagus, the diagnosis can be confirmed finally. Still some researchers think that if benign squamous cells, fungus, bacterial colonies, vegetable ingredients can be seen in chest drainage fluid, the diagnosis can also be made definitively. ${ }^{14}$

For the treatment of spontaneous esophageal rupture, most people think that it should be given operative treatment actively, especially if the onset time is within 24 hours. One-stage repair of the esophagus rupture can be done, at the same time food residue and necrotic tissue in thorax and mediastinal should be removed during the surgery, an unobstructed closed chest drainage should be kept after operation. ${ }^{15}$ Even some scholars believe that as long as the patients' physical condition allows, all should be given operative treatment actively without time constraints. ${ }^{16}$ Operation methods mainly include repair of esophageal rupture, resection of esophagus and reconstruction of esophagus with stomach, twostage esophageal resection and reconstruction of esophagus with colon, etc. Most patients admitted to hospital were within 24 hours in this article, the first 
three patients were given operative treatment, but there were two cases of recurrence of esophageal fistula after operation, complicated with thoracic and pulmonary infection, and eventually died of multiple organ failure. Probe into the causes of death, it might be due to vomiting after drinking, esophageal rupture let a large number of food residue containing alcohol flowing into mediastinum and chest through the rupture, and then lead to a series of physical and chemical reaction. The tissue surrounding the rupture become inflamed and edematous, and the release and infiltration of various inflammatory mediators changes the local internal environment. Even though early operative treatment if initiated it does not show results as the effect of local inflammatory mediators is initiated. Surgery can rectify the necrotized tissues, but correcting the change of internal environment can only depend on individual. If the surgery is done on time, it certainly will exacerbate the damage on the basis of original injury. On the one hand, the damage will increase the consumption of nutrition; and on the other hand it will increase the difficulty of the injury healing after the suture because of the exacerbated inflammatory edema of tissue. So although in principle, the sooner operation implemented the better results obtained, it is not suitable to everyone. For some special cases such as infirm or drunken people, choosing operative treatment should be cautious. Data shows that esophageal perforation can relapse after repair of esophageal rupture, but it can be recovered well only after conservative treatment, so the conclusion is that repair of esophageal rupture is not important for the whole treatment process, ${ }^{17}$ drainage of food stuffs and liquid in the esophagus is important.

As to the non-surgical treatment of spontaneous esophageal rupture, except for nutritional support, antiacid, anti-inflammatory treatment, the main methods include chest drainage, esophageal stent placement and endoscopic therapy, etc. The mode of treatment used was mainly chest drainage, with oral saline irrigation, and a good effect was obtained, except for one patient who gave up treatment and one case of encapsulated pyothorax who underwent surgery. Esophageal stent placement has a smaller injury compared with surgery, but study observed that endoscopic stent placement for patients with spontaneous esophageal rupture has no advantage in disease status, hospital stay, and it even has a higher failure rate which need operation, at the same time the mortality rate is higher than surgery. ${ }^{18}$ But some scholars have combined it with debridement and obtained a good effect. ${ }^{19}$ Therefore, it is may be that esophagus stent placement leads to inadequate drainage, and easily to remain mediastinal or chest infection. ${ }^{20}$
In a nutshell, spontaneous esophageal rupture is one of the thoracic surgery emergencies, and has an increasing trend in recent years. The disease is easy to be confused with heart disease and acute abdominal disease, so clinicians should identify it cautiously and try to prevent misdiagnosis and delayed treatment. As to treatment, we think that it should be given priority to conservative treatment, including unobstructed drainage and irrigation, keeping balance of nutritional condition and water-electrolyte, and a good effect can usually be achieved.

\section{REFERENCES}

1. Zhou Qiang. The experience of diagnosis and treatment of 40 cases of spontaneous esophageal rupture. World Health Digest 2013; 4(10): 132-133.

2. Sanka S, Gomez A, Heuschkel R and Krishnamurthy K. Boerhaave's syndrome: a differential diagnosis of acute chest pain following a vomiting illness. West Indian Med J 2013; 62(2):152-153.

3. Zhenyu $\mathrm{T}$ and Yongliang $\mathrm{Y}$. The experience of diagnosis and treatment of 17 cases of spontaneous esophageal rupture. Qinghai Medical Journal 2005; 35(3): 25-26.

4. Jacksona WE, Mehendiratta V, Palazzo J, DiMarino AJ, Quirk DM and Cohen S. Boerhaave's syndrome as an initial presentation of eosinophilic esophagitis: a case series. Annals of Gastroenterology 2013; 26: 166-169.

5. Vernon N, Mohananey D, Ghetmiri E and Ghaffari G. Esophageal Rupture as a Primary Manifestation in Eosinophilic Esophagitis. Case Reports in Medicine 2014 (2013): 673189.

6. Naitoh H, Fukuchi M, Kiriyama S, Fukasawa T, Tabe Y, Yamauchi $\mathrm{H}$, et al. Recurrent, spontaneous esophageal ruptures associated with antiphospholipid antibody syndrome: report of a case. Int Surg 2014; 99(6):842-845.

7. Thermann F, Thermann M and Dralle H. Therapy and course of disease in spontaneous esophageal perforations. ZentralbIChir 2006; 131(6):454-459.

8. Quanlin $M$, Xing $\mathrm{J}$ and Xuguag $\mathrm{Z}$. The experience of diagnosis and treatment about 75 cases of spontaneous rupture of esophagus. Chinese journal of ethnomedicine and ethnopharmacy 2013; 22(16): 102-104.

9. Gupta RK, Sah PL, Sah S, et al. Atypical presentation of Boerhaave's syndrome. BMJ Case Reports 2012: bcr2012006368.

10. Brændholt Rasmussen K and Grove MC. Boerhaave's syndrome can be a rare cause of tension pneumothorax. Ugeskr Laeger 2012; 174(38):2235-2236.

11. Tomiyama $\mathrm{K}$ and Yamamoto $\mathrm{T}$. Empyema from spontaneous esophageal rupture treated by thoracoscopic surgery. Kyobu Geka 2013; 66(11):1010-1013.

12. Garas G, Zarogoulidis P, Efthymiou A, et al. Spontaneous esophageal rupture as the underlying cause ofpneumothorax: early recognition is crucial. Thorac Dis 2014; 6(12):1655-1658.

13. Sato T, Obinata I, Takahashi S, Sasagawa M, Wanifuchi T, Sato I, et al. Spontaneous esophageal rupture successfully treated by conservative therapy: report of two cases. Surg Today 2002; 32(5):421-425.

14. Walid E. Khalbuss, Hooda S and Auger M. Cytomorphology of Boerhaave's syndrome: A critical value in cytology. Cyto Journal 2013; 10 : 8.

15. Wang Xingbang and Pan Yu. Clinical analysis and experience 
of 12 cases of spontaneous esophageal rupture surgery. An hui Medical and Pharmaceutical Journal 2014;18(1): 131-132.

16. Van Weyenberg SJ, Stam FJ and Marsman W. Successful surgical strategy in a late case of Boerhaave's syndrome (Boerhaave syndrome). Gastrointest Endosc 2014; 80(1):162.

17. Hu Yang, Yuan Yong and Zheng Xi. The treatment of spontaneous esophagus rupture.Chin J Gastrointest Surg 2014; 9(17): 934-936.

18. Schweigert $M$, Beattie $R$, Solymosi $N$, Booth $K$, Attila D,
Andrew M, et al. Endoscopic stent insertion versus primary operative management for spontaneous rupture of the esophagus (Boerhaave syndrome): an international study comparing the outcome. Am Surg 2013; 79(6):634-640.

19. Koivukangas V, Biancari F, Meriläinen S, et al. Esophageal stenting for spontaneous esophageal perforation. The Journal of Trauma Acute Care Surg 2012; 73(4):1011-1013.

20. Jansen JC, van Dop WA, Fockens P, Löwenberg M. Acute upper abdominal pain after excessive vomiting: Boerhaave's syndrome. Ned Tijdschr Geneeskd 2013;157(35):A6374.

\author{
Authors Contribution: \\ Involved in almost all the surgeries, gathered the statistics data, and wrote this essay.
}

Source of Support: Nil. Conflict of Interest: None. 